\title{
Unusual and dramatic presentation of "adem": what could be done in neurosurgical practice?
}

\begin{abstract}
The authors describe the case of a young patient who presented with new onset of acute disseminated encephalomyelitis responsible for progressive unilateral brain edema causing trans-tentorial herniation. The patient underwent urgent standard right hemispheric decompressive craniectomy; unfortunately this was not successful and the patient had rapid neurological demise.
\end{abstract}

Keywords: Acute disseminated encephalomyelitis, Cerebral edema, Hemicraniectomy
Volume 2 Issue 3 - 2015

\author{
Houssem Abid,' Jean-Etienne Herbrecht, ${ }^{2}$ \\ Marco Marsella, ${ }^{3}$ Jerome De Seze, ${ }^{4}$ Leonardo \\ Tigan,' Salvatore Chibbaro' \\ 'Neurosurgery Department, Hautepierre University Hospital, \\ France \\ ${ }^{2} \mathrm{CCU}$ Department, Hautepierre University Hospital, France \\ ${ }^{3}$ Neurosurgery Department, Gilbert, USA \\ ${ }^{4}$ Neurology Department, Hautepierre University Hospital, \\ France
}

Correspondence: Abid Houssem, Neurosurgery Department, Hautepierre University Hospital, Strasbourg, France,

Email abidhousseme@hotmail.com

Received: April 29, 2014 | Published: May 08, 2015
Abbreviations: ADEM,Acute disseminated encephalomyelitis; ICU, Intensive Care Unit; CT, Computed Tomography; MRI, Magnetic Resonance Imaging; ICP, Intracranial Pressure; CNS, Central Nervous System

\section{Introduction}

Acute disseminated encephalomyelitis (ADEM) is an inflammatory demyelinating immune-mediated disorder more common in the pediatric population. It is often associated with either viral or bacterial infection or it may be seen as rare post vaccination complication. It is characterized by an inflammatory reaction and demyelination of the central nervous system (CNS), with pathological changes occurring typically around small veins. ${ }^{1}$

The demyelination may occur anywhere in the brain, accounting for the wide spectrum of its clinical manifestations and disease severity. The pathophysiology involves transient autoimmune response directed to myelin or other self-antigens, or by nonspecific activation of auto-reactive $\mathrm{T}$ cell clones. ${ }^{2,3}$

Although some patients present with minor focal CNS abnormalities, others have a more rapid and extreme presentation. Acute clinical presentation is characterized by a rapid onset of encephalopathy, multifocal neurological deficits, convulsions, and impaired consciousness with fever. There are no biological markers of the disease.

Generally considered to be an autoimmune response against CNS myelin-associated antigens. ${ }^{4}$ the mainstay of medical treatment is focused on immunosuppression and supportive therapy. Although high-dose corticosteroids are most commonly used in the treatment of ADEM, other therapies have been used alone or in association including cyclophosphamide, intravenous immunoglobulin and plasmapheresis..$^{3-5}$ The indications for surgical treatment remain still controversial.

Rarely, ADEM may cause severe brain swelling responsible for intracranial hypertension. ${ }^{6}$ in case of brain swelling with raised intracranial pressure (ICP), refractory to conventional medical management, surgical decompression has been advocated as a "last resort form of treatment". ${ }^{6,7}$ The reversible nature of most of the deficits caused by ADEM justifies an aggressive management to avoid irreversible injury and death due to extensive brain swelling and its related consequences.

Brain and spinal MRI are the imaging modality of choice to point out white matter changes in ADEM. In this paper the authors describe how dramatic evolution of ADEM may be; also, relevant issues such as clinical features and possible management in neurosurgical practice along with pertinent literature will be addressed.

\section{Case report}

We report the case of a 32-year-old woman, with no relevant medical history, who presented with sudden onset of left hemiparesis following pneumonia. Following admission to the Neurological ward an urgent MRI showed an isolated right fronto-parietal lesion, hypointense on $\mathrm{T} 1$, hyperintense on $\mathrm{T} 2$ with mild mass effect on the surrounding brain structures as well as multiple micro- hemorrhagic lesions with peripheral contrast enhancement and a smaller second left frontal localization (Figure 1).

In the first instance the hypothesis of a pseudo-tumoral multiple sclerosis was considered and steroids were started. Follow up CT completed 24 hours later showed however increased mass effect on the right occipital horn although the patient seemed to do relatively well from a clinical standpoint. Corticoids were reduced (Figure 2).

Forty-eight hours later however the patient develop a sudden neurological deterioration and she was noted to be more somnolent; follow up brain MRI was repeated and showed significant enlargement of the previous lesion with relevant edema and midline shift. Higher steroids doses were administered and, once again good functional and clinical amelioration was noted (Figure $3 \& 4$ ).

At day 5 steroids were weaned off but within 24 hours, the patient started to complain of severe headache; in addition she became progressively drowsy and right mydriasis was noted as well; at this stage an urgent CT-scan showed massive right fronto-temporo- 
parietal edema with significant midline shift and right trans-tentorial herniation (Figure $5 \& 6$ ).

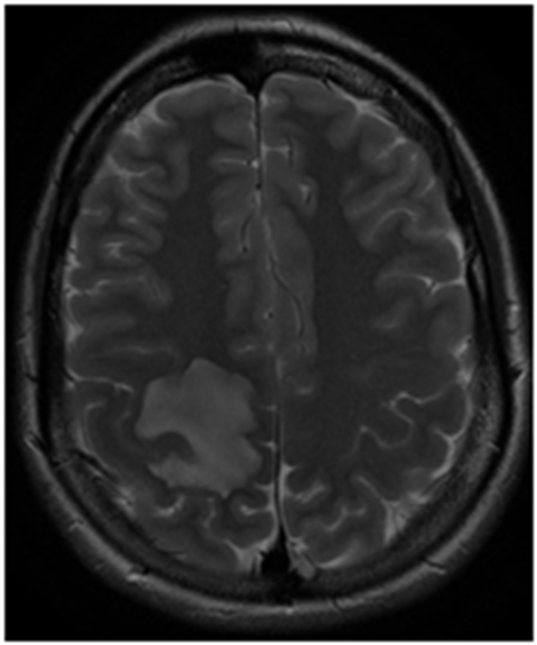

Figure I N=57; Epidemiological distribution of the pathological fractures, traumatic fractures, and nonunion.

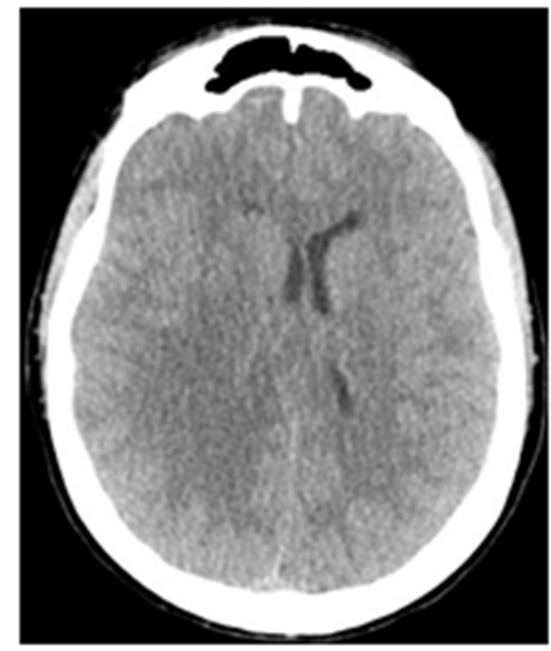

Figure 2 Axial non-enhanced CT scan (24 hours check) showing a right fronto-parietal edema with mass effect on lateral ventricle and midline shift.

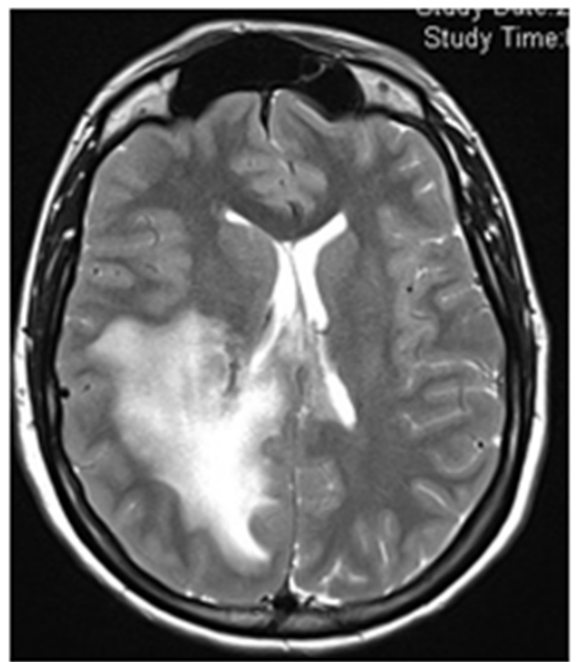

Figure 3 Axial T2 brain MRI showing extension of the known lesion (72 hours check).

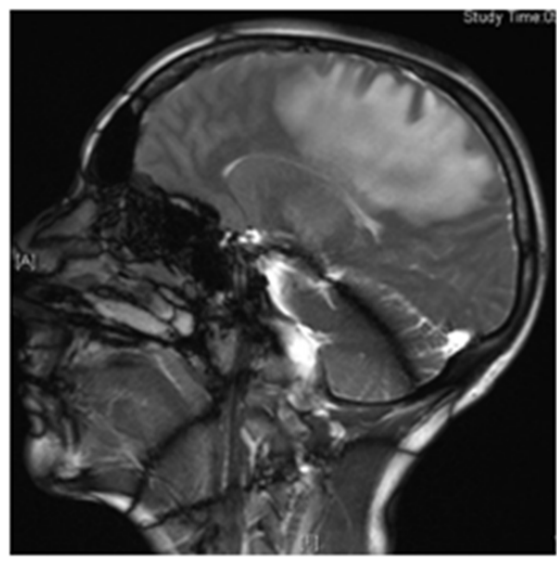

Figure 4 Sagittal T2 brain MRI showing large fronto-parietal lesion (72 hours check).

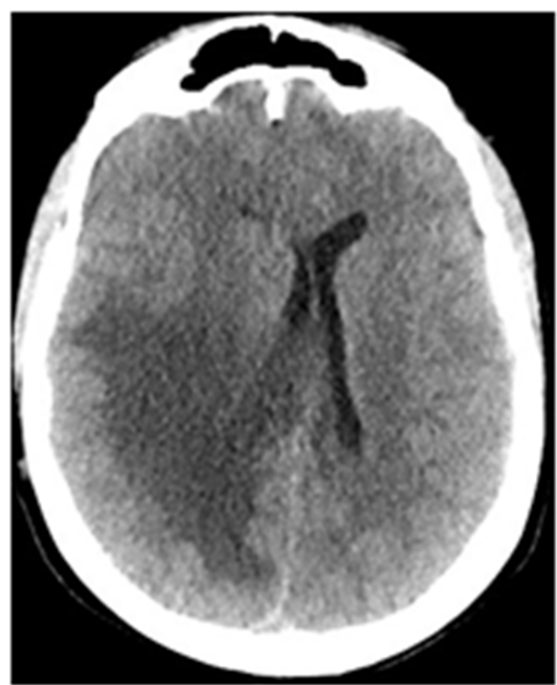

Figure 5 Axial non-enhanced CT scan showing massive brain edema with midline shift (I20 hour from admission).

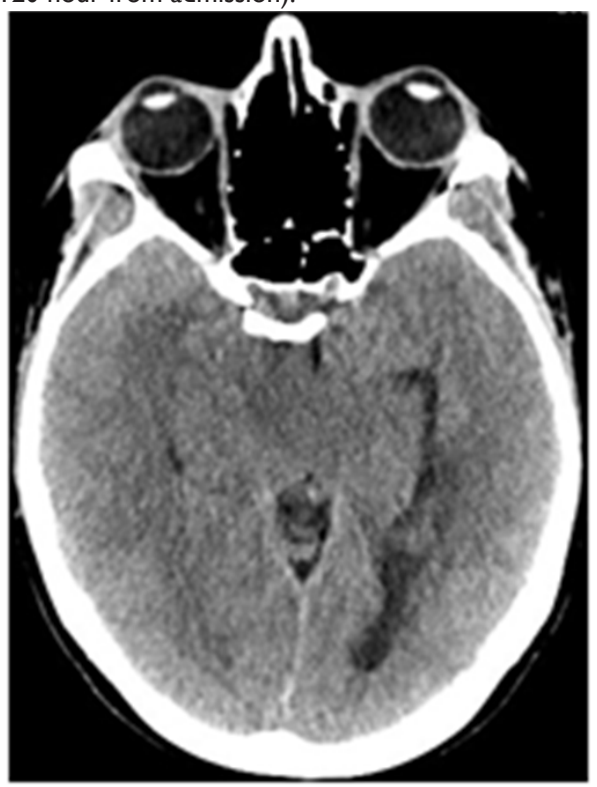

Figure 6 Right unco-temporal herniation with brain stem compression (I20 hour from admission). 
Her clinical condition rapidly deteriorated within 30 minutes and she developed bilaterally dilated unreactive pupils; she was then taken immediately on the operating room and underwent urgent decompressive right hemicraniectomy; the procedure allowed massive brain herniation rendering scalp closure very difficult. This actually required right frontal and temporal lobectomy as well as temporalis muscle amputation. Despite the procedure, the patient expired 36 hours after the operation. During surgery random biopsies were performed showing features in favor of ADEM, which was also been confirmed by autopsy.

\section{Discussion}

ADEM is an inflammatory immune-mediated condition more commonly observed in children after a common viral or bacterial infection or vaccination. ADEM is quite rare in adults and its differential diagnosis includes malignancy, infection as well as other demyelinating disorder. ${ }^{1,8}$ In 2007, the International Pediatric Multiple Sclerosis Study Group defined ADEM as "the first clinical event with a poly-symptomatic encephalopathy, with acute or subacute onset, showing focal or multifocal hyperintense lesions predominantly affecting the CNS white matter." This definition shows how difficult it may be to differentiate ADEM from a multiple sclerosis at an early stage. $^{8}$

Pathophysiology remains still unclear and it is probably due to a transient autoimmune response against myelin or other selfantigens. ${ }^{2,3}$ Brain biopsy is rarely done and performed in difficult and selected cases. ${ }^{9} 10$ Histological features of ADEM include perivenous demyelination and lymphocytic, plasma cells and monocytes vessel wall infiltration. ${ }^{11}$

Only few case of clinically aggressive forms of ADEM have been reported, thus knowledge about the best management of this condition is lacking. ${ }^{12}$ Although immunosuppressive therapy is the mainstay of treatment, the slow improvement leaves room for abrupt and malignant progression of cerebral edema within the first week of treatment, as it happened in our case. Our patient developed progressive and severe brain swelling with trans-tentorial herniation which did not respond to steroids and conservative treatment. In our interdisciplinary meeting the case was largely discussed and debated with neurologist.

Consensus was that maybe steroids were weaned off too early especially in consideration of the discrepancy between clinical and radiological findings; while the patient improved and relapsed twice from a clinical standpoint, CT and MRIs showed constant progression of the lesions indicating an extremely aggressive pathological process probably refractory to therapy.

Table I Reported cases in the English literature
From a diagnostic point of view CT scan represent a useful investigation showing usually white matter enhancing hypodense lesions which increase progressively in size. MRI remains however the gold standard imaging in suspected ADEM, although findings can vary a lot: Classically FLAIR sequences show multiple white matter, asymmetric hyperintense areas. ${ }^{8,13}$ more frequently involving thalami, basal ganglia, and periventricular white matter. ${ }^{14-16}$ Enhancement of T1-weighted images is not constant. ${ }^{8}$

Management of ADEM is currently well established. ${ }^{17}$ In the acute stage, it includes high-dose of intravenous steroids, intravenous immunoglobulin, and plasmapheresys ..$^{2}$ Corticosteroid as methylprednisolone is the first-line $(10-30 \mathrm{mg} / \mathrm{kg} / \mathrm{day}$, up to a maximum of $1 \mathrm{~g} /$ day). ${ }^{2}$ If aggressively treated, ADEM has a good prognosis and more than $50 \%$ of the patients achieve total functional recovery. ${ }^{18}$ while the remaining $50 \%$ may suffer mild neurological deficits ${ }^{2,18}$

In the majority of cases, increased ICP can be managed by conservative treatment, with a combination of the usual measures such as $30^{\circ}$ head elevation, mannitol therapy, hypertonic fluid infusion, brief and relative hyperventilation, and mild hypothermia. When ICP remains elevated despite conservative treatment, the remaining options may also include barbiturate coma and/or decompressive craniectomy.

Patient ICU admission with airway protection is always necessary. A crucial point is also an accurate management of the electrolytes and metabolism maintaining correct fluid balance. If seizures develop, specific anti-epileptic treatment is required. In case of failure of all available conservative treatment, surgical decompression of a swollen brain may represent the last resource. The potential benefits of this procedure in decreasing ICP and relieving/impending herniation with brainstem compression have been increasingly reported for stroke and trauma. Although timing and patient selection for decompression remain controversial, anecdotal case series and some prospective studies suggest that adults with brain swelling from large hemispheric infarctions may benefit from decompressive craniectomy. ${ }^{19-25}$

Mortality in ADEM varies from 1 to $12 \%$. $^{2,18,26,27}$ and refractory raised ICP remains the primary cause of death. Decompressive craniectomy in ADEM has been very rarely reported in the international literature and up-to-date only 5 cases are described in current literature to our knowledge. ${ }^{7,26,28-30}$ Interestingly it appears that all patients had a favorable outcome with a GOS of 4 or 5 (Table 1).

\begin{tabular}{|c|c|c|c|c|c|c|c|}
\hline Case no & Authors & Sex & Age Year/ Month & Symptoms & Duration & $\begin{array}{l}\text { History of } \\
\text { Recent Infection }\end{array}$ & Outcome \\
\hline $\mathrm{I}$ & Von Stucckrad, 2003 & 3 웅 & $34 y$ & Fever+headache & Since 6 days & No & Complete clinical recovery \\
\hline 2 & Daniel Refai, 2005 & $q$ & $51 y$ & $\begin{array}{l}\text { Subacute onset of fatigue+spatial } \\
\text { desorientation+headache+left } \\
\text { hemiparesis }\end{array}$ & Since 2 days & Yes & $\begin{array}{l}\text { Discharged home } 6 \text { weeks } \\
\text { after intervention }\end{array}$ \\
\hline 3 & Nilsson, 2009 & 운 & $50 y$ & $\begin{array}{l}\text { Partial epileptic } \\
\text { seizures+dysdiadochokinesis of her } \\
\text { right arm+dysphasia+right sided } \\
\text { facial palsy }\end{array}$ & Since 14 days & Yes & $\begin{array}{l}\text { After } 6 \text { weeks: very mild } \\
\text { dysphasia+slight paresis in } \\
\text { the right arm }\end{array}$ \\
\hline 4 & Ahmed, 2010 & q & $38 y$ & $\begin{array}{l}\text { Headache, vomiting, left sided } \\
\text { weakness }\end{array}$ & Since I week & Yes & $\begin{array}{l}\text { Discharged home on day II. } \\
3 \text { month after, no residual } \\
\text { neurological deficit }\end{array}$ \\
\hline 5 & Granget, 2012 & q & $18 \mathrm{~m}$ & Left hemibody seizures fever & Few hours & Yes & $\begin{array}{l}\text { Two years later, discrete right } \\
\text { hemiparesis }\end{array}$ \\
\hline 6 & Present case & q & $32 y$ & Left hemiparesis & Few hours & Yes & Dead \\
\hline
\end{tabular}


Pathological feature in the acute form of ADEM, which is also characterized by acute hemorrhagic leukoencephalitis, involves brain edema with numerous small hemorrhages. When cerebral edema is massive, this may cause temporal lobe and/or tonsillar herniation. Necrotic vascular lesions, focal demyelination and polynuclear infiltrates represent the main pathological findings; deposition of fibrin in vessel walls and in the Virchow-Robin spaces is a typical aspect.

ADEM usually develops one or two weeks following a viral disease or vaccination; its clinical presentation is characterized by fever, headache, vomiting and meningeal signs followed rapidly (in the most aggressive forms) by a combination of drowsiness, coma, seizures and focal, neurological signs. Recently, due to the progress of ICU care its prognosis has greatly improved. In a recent study about a cohort of 50 ADEM cases, an upper respiratory tract infection was evident in $50 \%$ of the patients. ${ }^{31}$ although transient cardiomyopathy and severe acute pulmonary edema have been reported in the acute stage. ${ }^{32}$

In the majority of ADEM cases, the cerebrospinal fluid shows only minor and unspecific changes. Cell count might be lightly increased accounting to the less than 10 cells $/ \mathrm{ml}$, with a total protein content also increased but usually below $100 \mathrm{mg} / \mathrm{dl}$. In many instances, the immunoglobulin level is also elevated but oligoclonal bands are seen only rarely. ${ }^{33,34}$ In any case, when ADEM is suspected, it is very important to avoid dangerous delay as its differential diagnosis is quite difficult especially in the acute stage.

Magnetic resonance imaging can help in differentiating ADEM from multiple sclerosis although many features look the same, as it was in our case. In ADEM, lesions are typically extensive but poorly defined. They are mainly found in the white matter but can extend to the deep gray matter. Multiple sclerosis lesions are commonly found in the periventricular white matter and corpus callosum and are less likely to involve the gray matter. ${ }^{1-3}$ Patients with monophasic ADEM should not have new lesions on follow-up imaging, whereas MS may be a relapsing disease. Diagnosis of ADEM is usually retrospective; reviewing the clinical presentation along with laboratory and progression seen in neuroimaging. Prognosis for ADEM is favorable, although $20 \%$ of patients develop recurring demyelination and will eventually progress later on to frank multiple sclerosis.

\section{Conclusion}

We describe a dramatic presentation of ADEM in which accurate diagnosis was misleading which, we feel, may have led to suboptimal clinical management. Salvage, yet more than occasionally, life-saving procedure was attempted as extreme resource. This was not successful very likely because of acute development of irreversible lesions and massive refractory hemispheric edema. Although medical treatment alone is usually very effective in the management of patients with ADEM, one should bear in mind that in case of refractory brain edema and raised ICP, decompressive craniectomy may represent a lifesaving procedure. Even though in our case this was ineffective, we should not forget that in the international literature, there is anecdotal evidence suggesting the role of decompressive craniectomy as an effective and safe treatment. ${ }^{35-37}$

\section{Ackowledgments}

None.

\section{Conflicts of interst}

None.

\section{References}

1. Menge T, Hemmer B, Nessler $\mathrm{S}$, et al. Acute disseminated encephalomyelitis: an update. Arch Neurol. 2005;62(11):1673-1680.

2. Alexander M, Murthy JM. Acute disseminated encephalomyelitis: Treatment guidelines. Ann Indian Acad Neurol. 2011;14(Suppl1):S60 S64.

3. Hynson JL, Kornberg AJ, Coleman LT, et al. Clinical and neuroradiologic features of acute disseminated encephalomyelitis in children. Neurology. 200156(10):1308-1312.

4. Hollinger P, Sturzenegger M, Mathis J, et al. Acute disseminated encephalomyelitis in adults: a reappraisal of clinical, CSF, EEG, and MRI findings. J Neurol. 2002;249(3):320-329.

5. Schwarz S, Mohr A, Knauth $M$, et al. Acute disseminated encephalomyelitis: a follow-up study of 40 adult patients. Neurology. 2001;56(10):1313-1318

6. Takata T, Hirakawa M, Sakurai M, et al. Fulminant form of acute disseminated encephalomyelitis: successful treatment with hypothermia. J Neurol Sci. 1999;165(1):94-97.

7. Von Stuckrad-Barre S, Klippel E, Foerch C, et al. Hemicraniectomy as a successful treatment of mass effect in acute disseminated encephalomyelitis. Neurology. 2003;61(3):420-421.

8. Tenembaum S, Chitnis T, Ness J, et al. Acute disseminated encephalomyelitis. Neurology. 2007;68(16 supp1 2):S23-S36.

9. Hashim HZ, Ibrahim NM, Wanyahya N, et al. A case of biopsy proven acute demyelinating encephalomyelitis (ADEM) with haemorrhagic leucoencephalitis. Ann Acad Med Singapore. 2011;40(4):197-200.

10. Hoche F, Pfeifenbring S, Vlaho S, et al. Rare brain biopsy findings in a first ADEM-like event of pediatric MS: histopathologic, neuroradiologic and clinical features. J Neural Transm. 2011;118(9):1311-1317.

11. Dale RC, de Sousa C, Chong WK, et al. Acute disseminated encephalomyelitis, multiphasic disseminated encephalomyelitis and multiple sclerosis in children. Brain. 2000;123(Pt 12):2407-2422.

12. Kinoshita A, Kaseda S, Yagi K, et al. A case of acute disseminated encephalomyelitis with pathologically-proven acute demyelinating lesion in the peripheral nervous system. Rinsho Shinkeigaku. 1994;34(9):892-897. [Article in Japanese].

13. Singh S, Alexander M, Korah IP. Acute disseminated encephalomyelitis: MR imaging features. AJR Am J Roentgenol. 1999;173(4):1101-1107.

14. Murthy SN, Faden HS, Cohen ME, et al. Acute disseminated encephalomyelitis in children. Pediatrics. 2002;110(2 Pt 1):e21.

15. Khurana DS, Melvin JJ, Kothare SV, et al. Acute disseminated encephalomyelitis in children: discordant neurologic and neuroimaging abnormalities and response to plasmapheresis. Pediatrics. 2005;116(2):431-436.

16. Khong $\mathrm{P}-\mathrm{L}$, Ho $\mathrm{H}-\mathrm{K}$, Cheng $\mathrm{P}-\mathrm{W}$, et al. Childhood acute disseminated encephalomyelitis: the role of brain and spinal cord MRI. Pediatr Radiol. 2002;32(1):59-66.

17. Rust RS. Multiple sclerosis, acute disseminated encephalomyelitis, and related conditions. Semin Pediatr Neurol. 2000;7(2):66-90.

18. Pavone P, Pettoello-Mantovano M, Le Pira A, et al. Acute disseminated encephalomyelitis: a long-term prospective study and meta-analysis. Neuropediatrics. 2010;41(6):246-255.

19. Carter BS, Ogilvy CS, Candia GJ, et al. One-year outcome after decompressive surgery for massive nondominant hemispheric infarction. Neurosurgery. 1997;40(6):1168-1176.

20. Delashaw JB, Broaddus WC, Kassell NF, et al. Treatment of right hemispheric cerebral infarction by hemicraniectomy. Stroke. 1990;21(6):874-881. 
21. Greenwood J. Acute brain infarctions with high intracranial pressure: surgical indications. Johns Hopkins Med J. 1968;122(5):254-260.

22. Ivamoto HS, Numoto M, Donaghy RM. Surgical decompression for cerebral and cerebellar infarcts. Stroke. 1974;5(3):365-370.

23. Kondziolka D, Fazl M. Functional recovery after decompressive craniectomy for cerebral infarction. Neurosurgery. 1988;23(2):143-147.

24. Rengachary SS, Batnitzky S, Morantz RA, et al. Hemicraniectomy for acute massive cerebral infarction. Neurosurgery. 1981;8(3):321-328.

25. Young PH, Smith KR, Dunn RC. Surgical decompression after cerebral hemispheric stroke: indications and patient selection. South Med J. 1982;75(4):473-474.

26. Ahmed AI, Eynon CA, Kinton L, et al. Decompressive craniectomy for acute disseminated encephalomyelitis. Neurocrit Care. 2010;13(3):393395.

27. Ketelslegers IA, Visser IE, Neuteboom RF, et al. Disease course and outcome of acute disseminated encephalomyelitis is more severe in adults than in children. Mult Scler. 2011;17(4):441-448.

28. Nilsson P, Larsson E-M, Kahlon B, et al. Tumefactive demyelinating disease treated with decompressive craniectomy. Eur J Neurol. 2009;16(5):639-642.

29. Refai D, Lee MC, Goldenberg FD, et al. Decompressive hemicraniectomy for acute disseminated encephalomyelitis: case report. Neurosurgery. 2005;56(4):E872; discussion E871.

30. Granget E, Milh M, Pech-Gourg G, et al. Life-saving decompressive craniectomy for acute disseminated encephalomyelitis in a child: a case report. Childs Nerv Syst. 2012;28(7):1121-1124.
31. Lin $\mathrm{C}-\mathrm{H}$, Jeng $\mathrm{J}-\mathrm{S}$, Hsieh $\mathrm{S}-\mathrm{T}$, et al. Acute disseminated encephalomyelitis: a follow-up study in Taiwan. $J$ Neurol Neurosurg Psychiatry. 2007;78(2):162-167.

32. Mawhinney E, Watt M, McDonnell GV. Transient cardiomyopathy as the presenting feature of acute disseminated encephalomyelitis. Mult Scler. 2009;15(12):1534-1536.

33. Stuve O, Nessler S, Hartung HP, et al. Acute disseminated encephalomyelitis. Pathogenesis, diagnosis, treatment, and prognosis. Nervenarzt. 2005;76(6):701-707.[Article in German].

34. Franciotta D, Columba-Cabezas S, Andreoni L, et al. Oligoclonal IgG band patterns in inflammatory demyelinating human and mouse diseases. J Neuro immunol. 2008;200(1):125-128.

35. Guresir E, Schuss P, Seifert V, et al. Decompressive craniectomy in children: single-center series and systematic review. Neurosurgery. 2012;70(4):881-889.

36. Jacob AT, Heuer GG, Grant R, et al. Decompressive hemicraniectomy for pediatric traumatic brain injury: long-term outcome based on quality of life. Pediatr Neurosurg. 2011;47(2):81-86.

37. Nguyen HS, Callahan JD, Cohen-Gadol AA. Life-saving decompressive craniectomy for diffuse cerebral edema during an episode of new-onset diabetic ketoacidosis: case report and review of the literature. Childs Nerv Syst. 2011;27(4):657-664. 